\title{
Die Vogelsiedlung des Neusatzer Riedes in Ungarn.
}

\section{Von Robert Berge, Zwickau i. S.}

Der einst vom linken Ufer der unteren Theiss in der Nähe der Stadt Titel weit nach Osten und im Süden bis an die Donau reichende sogenannte ,,weisse Morast," dem u. a. Baldamus, welcher ihn 1847 besuchte, seine bekannte Schilderung lieh, ist entwässert und in Ackerland umgewandelt worden, und das „Titeler Ried,“ das sich westlich der Theiss ausbreitete und etwa $100 \mathrm{qkm}$ umfasste, verfiel vor einigen Jahren demselben Schicksal. Denn über den marinen Niederschlagsschichten der Tertiärzeit ruhen in den Rieden, wie in der ungarischen Tiefebene überhaupt, diluviale und obenauf alluviale Ablagerungen, die nach dem Verschwinden des ehemaligen Meeres von den grossen Flussläufen als Zertrümmerungsprodukte aus den Gebirgen herabgeschwemmt wurden, zu bedeutender Mächtigkeit anstiegen und, in ihrer oberen Krume durch die abgestorbenen Reste der daraufwuchernden Pflanzendecke gesättigt, einen ausserordentlich fruchtbaren Kulturboden abgeben. Mit diesen Trockenlegungen gingen natürlich auch die grossartigen Vogelsiedlungen unter, welche jene Gegenden auszeichneten. Unweit davon besitzt indes noch die donauaufwärts gelegene Stadt Neusatz oder Ujvidek ein Ried von ungefähr fünf Quadratkilometer Ausdehnung, in welches zu gelangen mir auf meiner im Sommer 1901 nach Ungarn unternommenen Reise, und zwar am 30. Juli möglich ward. Dasselbe befindet sich 6 Kilometer nördlich von Neusatz, steht mit einem Donauarme, der sich unterhalb der Stadt von dem Hauptstrome linksseitig abzweigt, vermittels eines Kanals in Verbindung und stellt im wesentlichen einen vom Frühling bis zum Herbste unter Wasser gesetzten Rohrwald dar, in dessen westlichem Teile zwei Inseln liegen. Am südlichen Rande zieht sich Sumpfwaldung aus Eichen und Silberweiden hin, während die übrigen Grenzen an Felder stossen. Das Rohr (Arundo phragmitis) wird hier fingerdick, wächst zu 5 Meter empor und lässt nur Rohrkolben (Typha latifolia und angustifolia), sowie die Wasserschere (Stratiotes aloides) stellenweise in erheblicherer Menge aufsprossen, wogegen Sparganium, Glyceria, Salvinia natans u. s. w. sehr vereinzelt vorkommen. Im Winter wird das Wasser nach der Donau abgelassen und das Rohr für die Zwecke praktischer Verwendung geschnitten. Herr Kornel Szlavi in Neusatz, Mitglied der Genossenschaft, 
welche die Jagd in dem Riede gepachtet hat, besass die Güte, mir eine Fahrt in dasselbe bereitwilligst $\mathrm{zu}$ ermöglichen und die Führung zu übernehmen, um vor allen Dingen der in der Mitte vorhandenen, einen Flächenraum von 3-4 Hektar beanspruchenden Vogelniederlassung einen Besuch abzustatten, welche Liebenswürdigkeit um so dankbarer anzuerkennen war, als sich die zu bewältigenden Schwierigkeiten grade ganz besonders häuften. Denn einesteils hielt sich die Temperatur auf einer ungewöhnlichen Höhe, das Thermometer zeigte schon längere Zeit vom Morgen bis zum Abend über $30^{\circ} \mathrm{C}$ im Schatten, und im Riede mangelte zudem eine Beschattung. Die am Eingange anwesenden Fischer schlugen die Teilnahme ab, weil sie es wegen der Hitze nicht wagten, ins Ried zu fahren, und wir mussten daher allein eindringen. Sodann bereitete der eingetretene niedrige Wasserstand bedeutende Hindernisse, indem sich der Kahn auf grössere Strecken zwischen den weit hervorstarrenden, eng zusammengedrängten, kräftigen Blattrosetten der Wasserschere, die sonst untergetaucht sind, und später ausser durch das Rohr zwischen Rohrstoppeln durchwinden musste, über welche er bei höherem Wasser ebenfalls hinweggeschwommen wäre. Etwa 150 Meter von der Kolonie vermochten wir ihn in dem dichten Röhricht überhaupt nicht mehr fortzubringen und sahen uns genötigt, ihn $\mathrm{zu}$ verlassen, um das Ziel durchs Wasser watend $\mathrm{zu}$ gewinnen. $4 \frac{1}{2}$ Stunde hatten wir schweisstriefend alle Kräfte angespannt, den nur einige Kilometer messenden Weg zurückzulegen, ein Beweis, mit welcher Erfahrung die Riedvögel für ihre Sicherheit gesorgt hatten, denn unzugängliche Wildnis scheint auch in Ungarn das stichhaltigste Mittel zu bilden, sie vor der Vernichtung zu schützen.

Unter den Brutvögeln des Neusatzer Riedes fehlen zunächst Ardea alba, A. garzetta, Platalea leucerodia, Pelecanus onocrotalus, Phalacrocorax carbo, Larus, Haliaetus albicilla, Pandion haliaetus u. a. Ardea cinerea nistet in etwa 50 Paaren, aber mehr gegen den Rand zu. Auch Ardea purpurea strich bereits nicht weit vom Eingange her häufig mit dem bezeichnenden polternden Geräusche von den Bäumen ab, obwohl auch das eigentliche Koloniegebiet eine Anzahl seiner Horste enthielt. Dagegen waren in letzterem namentlich Plegadis falcinellus, Ardea ralloides, Nycticorax griseus und Phalacrocorax pygmaeus zu erblicken. Ausserdem wurden im Riede bemerkt: Rallus aquaticus, Anser cinereus, Buteo vulgaris, 
Circus aeruginosus. Hydrochelidon nigra soll ebenfalls hier nisten, war jedoch zur Zeit meiner Anwesenheit schon davongezogen. Von Meisen scheint Panurus biarmicus und in dem anliegenden Walde Aegithalus pendulinus zu brüten. Totanus calidris und Numenius arcuatus liessen sich gleichfalls vernehmen, ohne daselbst zu nisten, und Botaurus stellaris, Ardetta minuta, Fulica atra, Gallinula chloropus, Lappentaucher, Enten und manche anderen erfordern bei ihrem selbstverständlichen Vorkommen wohl weiter keine besondere Erwähnung. Die Anzahl der Brutpaare schwankt wie überall je nach den verschiedenen Jahren, doch hat Herr Szlavi folgende Schätzung ermittelt, die als Anhalt für die Häufigkeit im Riede mitgeteilt sei: Ardea purpurea 150, A. ralloides 150-200, Nycticorax griseus 300, Plegadis falcinellus 100-200, Anser cinerea 10, Phalacrocorax pygmaeus 10-15 und Hydrochelidon nigra 10-50 Paare. Durch unsere Annäherung bereits in Aufruhr gebracht, bot die Kolonie ein seltnes Schauspiel, indem die aufgeregten Vögel eifrig hin und her schwärmten Verhältnismässig die geringste Scheu bekundeten die Sichler, die trotz der Schüsse immer wieder und oft nicht hoch über uns hinflogen, obwohl sie uns bei jedem neuen Anzug ins Auge gefasst haben mussten, wie man bei der Nähe deutlich zu erkennen vermochte. Das Gewehr schien sie sehr zu schrecken, denn sobald es erhoben wurde, prallten sie rasch nach oben oder lenkten nach der Seite $a b$, ohne indessen ganz umzukehren, während unser blosser Anblick ihre Richtung zuweilen kaum veränderte. Ihr Flugbild war gekennzeichnet durch die grossen, breiten Flügel, welche gegen 1 Meter klafterten, die weit nach hinten ragenden Beine, den langen, im Gegensatz zu den Reihern vorgestreckten Hals und den fast sichelförmig gekrümmten, langen Schnabel. Den Namen „Schwarzschnepfe“ verdienen sie insofern, als schon bei geringer Entfernung ihre Farben nicht mehr hervortreten, sondern sich zu Schwarz verwischen. Das Fliegen geschah mässig schnell und mit nicht weit ausholenden Schlägen, wobei die bekannte Fluganordnung eigentümlich auffiel, die sich in eine fast schnurartige, zwischen den Flügelspitzen der einzelnen Vögel nur kleine Räume freilassende Querlinie gliedert, welche nicht grade verläuft, sondern in beständigen Biegungen und Schwenkungen vorwärts schreitet. Eine merkwürdige Schnabelfärbung wies ein erlegtes junges Stück auf, dessen ungefähre Alterstufe vorerst durch folgende Angaben näher bestimmt werden möge: 
Körper von der Grösse einer Saatkrähe; Kopf, Hals, Unterleib und Füsse schwarz; Rücken vom Hinternacken an, Steuerfedern, obere Schwanzdecke und Flügel glänzend dunkelgrün mit Bronzeschimmer. Um den schwärzlichen Schnabel aber liefen zwei scharf abstechende, bläulichgraue Querbänder, und zwar so, dass Grund, Mitte und Spitze dunkel blieben, während selbst die neue Auflage von Naumanns Naturgeschichte der Vögel Mitteleuropas sagt: „Im Jugendkleide hat der dunkelfarbige Sichler einen einfarbig graugrünen Schnabel." Ob jene Bindenfärbung als Übergangsglied vom Schnabel der Nestjungen, der bekanntlich ein oder zwei gelbe Bänder trägt, angesprochen werden darf und allgemein auftritt, lässt sich auf den angeführten Einzelfall hin leider nicht entscheiden.

Am leichtesten und gewandtesten unter den im Ried vertretenen Reihern flog Ardea ralloides, welcher, den Hals in das lockere Gefieder zurückgelegt, in der Mitte ziemlich dick, hinten und vorn aber durch Füsse und Schnabel beinah zugespitzt erschien, wohingegen an Enten der Flug von Phalocrocorax pygmaeus, der ja auch in der Grösse etwa mit Anas querquedula übereinstimmt, erinnert. Auch Nycticorax griseus segelte vorbei, die gebogenen, nicht vollständig ausgestreckten Flügel von über ein Meter Spannbreite unhörbar und wenig rasch schwingend, den Hals kurz und dick zusammengezogen, mit hintergelegten, aber auffällig kurz aussehenden Beinen. Von Stimmen erschallte am häufigsten diejenige des Purpurreihers, die bekanntlich etwas mattem Fischreiherruf, oft auch, und besonders aus der Ferne, dem "Quak" der männlichen Stockente täuschend gleicht, wozu die übrigen ebenfalls Laute hervorstiessen. Ardea purpurea benahm sich übrigens vorsichtiger und kam niemals in Schussweite heran.

Die zahlreichen, oft nur eine Fusslänge getrennten Horste waren um diese Jahreszeit verödet, doch gut erhalten, und diejenigen des Purpurreihers, welche sehr umfänglich und, wie alle andern an der besuchten Stelle, auf umgeknickten Rohrstengeln inmitten von Rohrbüscheln errichtet waren, hielten unsere Körperlast vollständig aus, so dass sie als Ruhesessel benutzt werden konnten. Die Nester setzten sich, insoweit sie mir zu Gesicht kamen, aus Rohrteilen zusammen, ohne Beimengung von Reisern, und nur beim Sichler lagen in der Mulde dünne Weidenzweige. Der Aufbau zeigte das übliche Schema: unten gröbere und stärkere, nach oben hin feinere, weiche Stoffe, besonders Blätter; die Mulde war 
wenig vertieft und die Randumwallung ganz niedrig. Ihr senkrechter Abstand vom Wasserspiegel betrug etwa 0,5 bis 1 Meter, in welch letzterer Höhe namentlich die beträchtlich kleineren Horste des Rallenreihers gefunden wurden. Ein zurückgebliebenes und von Herrn Szlavi aufgefischtes $\mathrm{Ei}$ von Plegadis falcinellus besass die Grösse eines kleinen Hühnereies und das bekannte blaugrüne, der Rohrumgebung angepasste Kolorit. Wie der Alarm bewies, verwendeten die Kolonievögel das Ansiedlungsrevier mit ihren offenbar schon längere Zeit flugfähigen Jungen noch als Ruhestätte, und trotz unserer Nähe fielen sie, anscheinend empfindlich gegen die Hitze, welche in der Sonne sicherlich $40^{\circ} \mathrm{C}$ betrug, immer wieder in dasselbe ein, sodass mitunter ein Schuss abgefeuert werden musste, um die Bewegung aufs neue zu beflügeln. Bei der sich niedersenkenden Abendkühle schienen sie aufzuleben, und als wir auf dem Rückwege begriffen waren, zogen öfters Scharen oder einzelne aus der Richtung des Brutplatzes über uns dahin, wahrscheinlich, um sich nach den Futterstellen zu begeben.

\section{Über Grönlands Vogelwelt.}

Vortrag, gehalten vor der Deutschen Ornithologischen Gesellschaft am 7. Oktober 1901.

\section{Von Dr. O. Helms, Haslev.}

\section{Meine Herren!}

Wenn ich heute die Ehre haben soll, Ihnen etwas von den Vögeln Grönlands zu erzählen, so muss ich mir erlauben, ein paar Bemerkungen über das Land, seine Natur und das Bewohnen d esselben vorauszuschicken. Grönlands Lage ist Ihnen Allen bekannt, ebenso wie Sie wissen, dass es eine ungeheuere Insel von gegen 20,000 Quadratmeilen ist, wovon der weit überwiegende Teil mit einer mächtigen Eisdecke, dem Inlandseise, bedeckt ist, welches an einzelnen Stellen ganz bis zur Küste hinabreicht, sonst nur einen breiteren oder schmaleren Streifen freien Küstenlandes übrig lässt. Auf dieser schmalen Küste in einer Breite von höchstens 20 Meilen, oftmals viel weniger, ist es, wo sich alles Menschen-, Tier- und Pflanzenleben in Grönland befindet. Gewiss haben verschiedene Reisende Vögel auf dem Inlandseise 


\section{$2 \mathrm{BHL}$ Biodiversity Heritage Library}

1902. "Die Vogelsiedlung des Neusatzer Riedes in Ungarn." Journal fu

r Ornithologie 50, 87-91. https://doi.org/10.1007/bf02286797.

View This Item Online: https://www.biodiversitylibrary.org/item/104733

DOI: https://doi.org/10.1007/bf02286797

Permalink: https://www.biodiversitylibrary.org/partpdf/142578

\section{Holding Institution}

Smithsonian Libraries

\section{Sponsored by}

Biodiversity Heritage Library

\section{Copyright \& Reuse}

Copyright Status: Public domain. The BHL considers that this work is no longer under copyright protection.

This document was created from content at the Biodiversity Heritage Library, the world's largest open access digital library for biodiversity literature and archives. Visit BHL at https://www.biodiversitylibrary.org. 